\title{
Estudo etnobotânico de plantas medicinais para problemas bucais no município de João Pessoa, Brasil
}

\author{
Esther Bandeira Santos, ${ }^{1}$ Gesira Soares Dantas, ${ }^{1}$ Hosana Bandeira Santos, ${ }^{2}$ Margareth F. F. \\ Melo Diniz, ${ }^{2}$ Fabio Correia Sampaio ${ }^{*, 1}$
}

\author{
${ }^{1}$ Laboratório de Biologia Bucal, Departamento de Odontologia Clínica e Social, Universidade Federal da \\ Paraíba, 58051-900 João Pessoa-PB, Brasil, \\ ${ }^{2}$ Laboratório de Tecnologia Farmacêutica, Departamento de Ciências Farmacêuticas, Universidade Federal da \\ Paraíba, Caixa Postal 5009, 58051-900 João Pessoa-PB, Brasil
}

\begin{abstract}
RESUMO: O objetivo desta pesquisa foi realizar um estudo etnobotânico sobre a indicação de plantas medicinais para tratamentos de patologias bucais, bem como investigar sobre o uso de plantas medicinais entre usuários de serviços odontológicos na cidade de João Pessoa, Brasil. O levantamento consistiu em duas etapas de investigação: raizeiros $(n=20)$ e usuários $(n=$ 200). Foi aplicado um formulário aos raizeiros a fim de saber quais plantas medicinais são mais comercializadas para problemas bucais. Posteriormente, foi aplicado um formulário dirigido aos usuários de serviço público $(\mathrm{n}=100)$ e particular $(\mathrm{n}=100)$ com o objetivo obter informações sobre a utilização de fitoterápicos. As plantas de uso odontológico mais vendidas pelos raizeiros foram: babatenon (Pithecelobium avaremotemo Mart.), aroeira (Schinus terebinthifolius Raddi), cajueiro roxo (Anacardium occidentale L.) e quixaba (Bumelia sartorum Mart.). Dentre os usuários, 80\% afirmam utilizar plantas medicinais, sendo as mais citadas: a romã (Punica granatum L.), cajueiro roxo, juá (Zizyphus joazeiro Mart.), e hortelã de folha graúda (Plectrantus amboinicus Lour). Os dados sugerem que boa parte da população local, principalmente serviço público, faz uso de plantas medicinais, sendo necessária a realização de mais estudos que possam comprovar cientificamente a ação das mesmas.
\end{abstract}

Unitermos: Etnobotânica, plantas medicinais, fitoterapia, saúde bucal.

\begin{abstract}
Etnobotanical studies of medicinal plants for oral conditions in the municipality of João Pessoa, Brazil". The objective of this research was to carry out an ethnobotanical study about the indication of medicinal plants for oral conditions, as well as to investigate the use of medicinal plants among users of dental services in the city of João Pessoa, Brazil. The work consisted of two stages of investigation: traditional healers ("raizeiros") (n= $20)$ and users $(n=200)$. A structured form was applied to the healers in order to know which medicinal plant they marketed most for oral pathologies. Later, a form was applied to the users of public services $(n=100)$ and private services $(n=100)$ to obtain information about the use phytotherapy. The most frequent plants used for oral problems that were sold by the healers were: "babatenon" (Pithecelobium avaremotemo Mart.), "aroeira" (Schinus terebinthifolius Raddi), cashew tree known as "cajueiro roxo" (Anacardium occidentale L.) and "quixaba" (Bumelia sartorum Mart.). Among the users, $80 \%$ reported the use of medicinal plants. The most cited were pomegranate (Punica granatum L.), cashew tree, "juá" (Zizyphus joazeiro Mart.), and mint leaf known as "hortelã da folha graúda" (Plectrantus amboinicus Lour). The data suggest that a good deal of the local population, mainly public service users do use medicinal plants. It is necessary, though more studies to prove the effective action of the plants under scientific basis.
\end{abstract}

Keywords: Ethnobotany, phytotherapy, medicinal plants, oral health.

\section{INTRODUÇÃO}

O Brasil possui uma das maiores diversidades vegetal do mundo e inúmeras experiências vinculadas ao conhecimento popular das plantas medicinais e tecnologia para correlacionar o saber popular e científico (Coutinho et al., 2004; Albuquerque \& Hanazaki, 2006). No contexto do uso de plantas medicinais, a prática da fitoterapia vem recebendo amparo legal significativo nos últimos anos (Ribeiro et al., 2005; Silva et al., 2006; Carvalho et al., 2008; Marliére et al., 2008; Veiga-Junior, 2008). No município de João Pessoa foi aprovada a Lei Municipal 7.630 de 15/07/94, para a implantação do Programa de Plantas Medicinais, gerenciado pela Secretaria Municipal de Saúde (Semanário Oficial, 1994). Recentemente, o Ministério da Saúde, através da Portaria ${ }^{\circ} 971$ de 3 de maio de 2006, aprovou a Política Nacional de Práticas Integrativas e Complementares 
(PNPIC) no Sistema Único de Saúde onde se insere a fitoterapia (Brasil, 2006).

Do ponto de vista dos usuários dos serviços de saúde, as plantas medicinais são vistas na maioria das vezes apenas como uma alternativa aos elevados custos dos medicamentos convencionais e não como uma opção terapêutica devido às propriedades curativas das plantas (Ness et al., 1999). Na perspectiva dos profissionais de saúde, o desconhecimento sobre as indicações e cuidados no uso de plantas medicinais ainda é um problema (Oliveira et al., 1997). Apesar das inúmeras possibilidades de uso de plantas medicinais por parte dos profissionais da área da saúde, o uso de plantas medicinais na odontologia tem sido pouco explorado, seja para tratar doenças bucais ou para tratar doenças sistêmicas com manifestações bucais (Oliveira et al., 2007; Soyama, 2007; Lustosa et al., 2008). Xavier et al. (1995) identificaram pouco mais de 260 plantas medicinais distribuídas para cerca de 19 diferentes indicações para uso em odontologia. No entanto, plantas para uso na odontologia são relatadas pela população, mas poucas são avaliadas cientificamente quanto às suas propriedades biológicas. $\mathrm{O}$ avanço maior neste sentido está entre as plantas com atividade antimicrobiana e antifúngica pelo considerável avanço do desenvolvimento de derivados naturais com ação bactericida ou fungicida (Cowan, 1999; Vasconcelos et al., 2006; Silva et al., 2008; Vasconcelos et al., 2008).

Diante dos poucos trabalhos etnobotânicos voltados para o uso de plantas medicinais na odontologia, este artigo teve como objetivo identificar as plantas medicinais para problemas da cavidade bucal, mais indicadas por raizeiros e utilizadas por usuários de clínicas odontológicas em João Pessoa.

\section{MATERIAL E MÉTODOS}

Foi realizado um levantamento sobre o uso popular de plantas medicinais com indicação terapêutica na odontologia. Este levantamento foi realizado em duas frentes: raizeiros $(\mathrm{n}=20)$ e usuários $(\mathrm{n}=200)$. Para coleta dos dados foi utilizado um formulário para os raizeiros e outro para os usuários, com perguntas fechadas e abertas.

Foram entrevistados raizeiros que comercializam plantas medicinais nos mercados públicos da cidade de João Pessoa (Mercados de Oitizeiro, Central, Jaguaribe, Bairro dos Estados, Torre e Mangabeira). Estes foram entrevistados sobre os produtos e suas indicações da mesma forma como a informação é repassada para a população.

Em seguida, foi aplicado formulário aos usuários pacientes da Unidade Básica de Saúde Timbó I, pacientes de clínicas da UFPB (Universidade Federal da Paraíba) e uma clínica particular (Odontológica). O formulário objetivou traçar o perfil do indivíduo e também obter informações sobre as formas de como estes utilizam as plantas medicinais.

O projeto foi aprovado pelo comitê de bioética do Centro de Ciências da Saúde da UFPB. As entrevistas entre os raizeiros e usuários só foram realizadas após assinatura do termo de consentimento livre e esclarecido.

A partir das informações fornecidas pelos raizeiros e usuários foi identificada a relação de plantas de maior prevalência de uso em tratamentos bucais.

Os dados foram tabulados em programa SPSS v. 10.0. A análise estatística foi basicamente do tipo descritiva com apresentação de dados percentuais de freqüência. Quando apropriado observou-se correlação entre variáveis através de correlação de Pearson e teste de qui-quadrado com nível de significância de $5 \%$.

\section{RESULTADOS E DISCUSSÃO}

Os dados obtidos através das entrevistas dos raizeiros locais comprovam o uso de diversas plantas medicinais para fins odontológicos. Os raizeiros entrevistados apresentaram idade média de 50 anos (10 homens e 10 mulheres). O conhecimento em plantas medicinais foi adquirido, em sua maioria, através de familiares e livros. Trinta por cento concluíram o ensino médio. Quinze por cento tiveram ensino fundamental completo e $10 \%$ eram iletrados. Os entrevistados afirmaram que $75 \%$ das plantas vendidas são oriundas do interior do Estado.

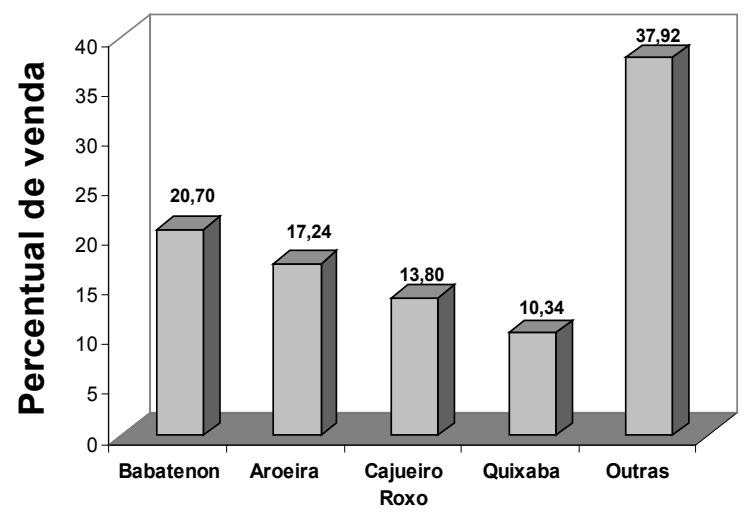

Figura 1. Plantas medicinais de uso odontológico mais vendidas em João Pessoa.

As plantas de uso odontológico mais vendidas pelos raizeiros foram: babatenon (Pithecelobium avaremotemo Mart.), seguida da aroeira (Schinus terebinthifolius Raddi), cajueiro roxo (Anacardium occidentale L.) e quixaba (Bumelia sartorum Mart.) (ver Figura 1). A mais indicada para halitose e dor de dente foi o cravo (Syzygium aromaticum L.); para afta ou ferida na boca, pedra ume (Myrcia salicifolia DC); e para sangramento gengival: babatenon, juá (Zizyphus 
Tabela 1. Plantas medicinais utilizadas por usuários do município de João Pessoa.

\begin{tabular}{|c|c|c|c|c|c|c|c|c|c|c|}
\hline \multirow[b]{2}{*}{ Planta } & \multicolumn{2}{|c|}{ Romã } & \multicolumn{2}{|c|}{ Cajueiro } & \multicolumn{2}{|c|}{ Juá } & \multicolumn{2}{|c|}{ Hortelã } & \multicolumn{2}{|c|}{ Aroeira } \\
\hline & $\mathrm{n}$ & $\%$ & $\mathrm{n}$ & $\%$ & $\mathrm{n}$ & $\%$ & $\mathrm{n}$ & $\%$ & $\mathrm{n}$ & \\
\hline Usa & 138 & 69,0 & 86 & 43,0 & 61 & 30,5 & 57 & 28,5 & 54 & 27,0 \\
\hline Não usa & 62 & 31,0 & 114 & 57,0 & 139 & 69,5 & 143 & 71,5 & 146 & 73,0 \\
\hline Total & 200 & 100,0 & 200 & 100,0 & 200 & 100,0 & 200 & 100,0 & 200 & 100,0 \\
\hline
\end{tabular}

Tabela 2. Forma de utilização e indicação mais citadas das plantas medicinais.

\begin{tabular}{lllll}
\hline Planta & N & $\%$ & Forma de uso & Indicação \\
\hline Romã (Punica granatum) & 138 & 86,25 & gargarejo & garganta \\
Cajueiro (Anacardium occidentale) & 86 & 53,75 & beber a infusão & inflamação \\
Juá (Zizyphus joazeiro) & 61 & 38,12 & escovação & limpar os dentes \\
Hortelã (Plectrantus amboinicus) & 57 & 35,62 & beber a infusão & tosse \\
Aroeira (Schinus terebinthifolius) & 4 & 33,75 & beber a infusão & inflamação \\
\hline
\end{tabular}

Tabela 3. Relação entre o uso de plantas medicinais em usuários do serviço público e particular do município de João Pessoa.

\begin{tabular}{lcccc}
\hline \multirow{2}{*}{ Serviço } & \multicolumn{3}{c}{ Tratamento com planta } & Total \\
\cline { 2 - 5 } & sim & não & 100 \\
Público & 86 & 14 & 100 \\
Privado & 74 & 26 & 200 \\
\hline Total & 160 & 40 & 200 \\
\hline
\end{tabular}

Qui-quadrado $=4,5 ;$ g.1. $=1 ; \mathrm{p}=0,03$.

joazeiro Mart.) e pedra ume. Dos entrevistados, 10 a $15 \%$ não sabiam indicar alguma planta para tais afecções.

Entre os usuários entrevistados $(n=200)$, podemos afirmar que $65 \%$ são do gênero feminino e mais da metade da população entrevistada é natural da capital, com faixa etária de 12 a 80 anos. A renda mensal de $62 \%$ dos entrevistados chega até dois salários mínimos. Dos 200 entrevistados, 160 fazem uso de planta medicinal para uso odontológico, sendo que 59\% fazem mistura de plantas medicinais com outras plantas, e $21 \%$ planta medicinal com medicamento alopático. Apenas um entrevistado (5\%) dos 160, foi recomendado a usar plantas medicinais por dentista, e $54,4 \%$ por amigos. Vinte e dois e meio por cento informam ao dentista quando usam plantas medicinais.

A planta mais citada foi a romã (Punica granatum L.) com $69 \%$ de relatos seguido do cajueiro roxo $(43 \%)$, do juá $(30,5 \%)$, da hortelã de folha graúda (Plectrantus amboinicus Lour) com 28,5\%, dentre outras, como mostra a tabela 1 . Os dados sobre as formas de uso e indicações mais citadas para estas plantas estão na Tabela 2.

Observou-se que a romã foi a mais citada pelos usuários dos dois tipos de serviços. Não houve diferença estatisticamente significativa quanto à freqüência no uso de plantas medicinais entre os usuários de serviço público e particular na cidade de João Pessoa (Tabela 3).

Através dos dados obtidos com os raizeiros, as plantas mais relatadas para uso em afecções bucais são conhecidas pela atividade analgésica (cravo), cicatrizante e antiinflamatória (babatenon, aroeira, cajueiro e quixaba). Já pelos usuários, as plantas mais citadas foram: romã, cajueiro roxo, juá, hortelã da folha graúda e aroeira; não havendo concordância entre os relatos dos raizeiros e dos usuários. Este último dado sugere que os usuários adquirem as plantas medicinais de outras formas. Além disso, sabe-se que atualmente a maioria da população, principalmente em áreas urbanas, utiliza plantas cultiváveis porque são mais acessíveis (Gazzaneo et al., 2005).

De acordo com o relato dos usuários, apenas um cirurgião dentista indicou o uso de plantas medicinais para fins de saúde bucal. Diante dos resultados do nosso estudo que comprovam o uso de plantas medicinais por parte dos usuários de serviços odontológicos se torna necessário a capacitação dos profissionais da área odontológica quanto ao uso de plantas medicinais. Adicionalmente, a aprovação da Política Nacional de Práticas Integrativas e Complementares (PNPIC) no Sistema Único de Saúde confirma a importância destes profissionais do SUS conhecerem e indicarem plantas medicinais para suprir as necessidades da população.

Agra et al. (2007, 2008) observaram que 483 espécies de plantas com propriedades ativas são utilizadas na região Nordeste. Apesar do número expressivo de plantas medicinais em uso, para a maioria delas, ainda não foram desenvolvidos estudos científicos sobre os seus constituintes ativos. Do mesmo modo os estudos sobre a comprovação científica da eficácia de plantas medicinais para problemas bucais ainda são escassos. Há poucos estudos pré-clinicos e clínicos específicos na odontologia. Vale salientar que o ambiente bucal com características de clearance salivar, biofilmes dentais e estruturas de tecido mole e duro exigem técnicas elaboradas e específicas (Singh et al., 2007). Por este motivo, pesquisas com base no 
conhecimento popular sobre uso de plantas medicinais para problemas bucais devem ser realizadas em outras localidades no sentido de avaliar diferenças regionais e identificar plantas medicinais com potencial para uso comprovado e seguro na odontologia.

\section{CONCLUSÕES}

Conclui-se que plantas medicinais para fins odontológicos são comercializadas em diversos bairros da cidade de João Pessoa. Os dados sugerem o uso de plantas medicinais por boa parte da população local principalmente no serviço público. A romã (Punica granatum L.) indicada para infecções bucais foi a planta mais citada pelos usuários dos dois tipos de serviços. As plantas mais comercializadas são, na maioria dos casos, nativas da flora nordestina e do próprio Estado.

\section{REFERÊNCIAS}

Agra MF, Freitas PF, Barbosa-Filho JM 2007. Synopsis of the plants known as medicinal and poisonous in Northeast of Brazil. Rev Bras Farmacogn 17: 114140.

Agra MF, Silva KN, Basílio IJLD, França PF, Barbosa-Filho JM 2008. Survey of medicinal plants used in the region Northeast of Brazil. Rev Bras Farmacogn 18: 472-508.

Albuquerque UP, Hanazaki N 2006. As pesquisas etnodirigidas na descoberta de novos fármacos de interesse médico e farmacêutico: fragilidades e pespectivas. Rev Bras Farmacogn 16 (Supl.): 678-689.

Brasil 2006. Decreto $\mathrm{n}^{\mathrm{o}} 5.813$ de 22 de junho de 2006. Aprova a Política Nacional de Plantas Medicinais e Fitoterápicos e dá outras providências. Diário Oficial da União, Brasília, DF, jun. 2006.

Carvalho ACB, Balbino EE, Maciel A, Perfeito JPS 2008. Situação do registro de medicamentos fitoterápicos no Brasil. Rev Bras Farmacogn 18: 314-319.

Coutinho HDM. Bezerra DAC, Lôbo K, Barbosa IJF 2004. Atividade antimicrobiana de produtos naturais. Conceitos 5: 77-85.

Cowan MN 1999. Plant products as antimicrobial agents. Clin Microbiol Rev 12: 564-82.

Gazzaneo LRS, Lucena RFP, Albuquerque UP 2005. Knowledge and use of medicinal plants by local specialists in an region of Atlantic Forest in the state of Pernambuco (Northeastern Brazil). J Ethnobiol Ethnomed 1: 1-8.

Lustosa SR, Galindo AB, Nunes LCC, Randau KP, Rolim Neto PJ 2008. Própolis: atualizações sobre a química e a farmacologia. Rev Bras Farmacogn 18: 447-454.

Marliére LDP, Ribeiro AQ, Brandão MGL, Klein CH, Acurcio FA 2008. Utilização de fitoterápicos por idosos: resultados de um inquérito domiciliar em Belo Horizonte (MG), Brasil. Rev Bras Farmacogn 18 (Supl.): 754-760.

Ness J, Sherman FT, Pan CX 1999. Alternative medicine: What the data say about common herbal therapies. Geriatrics 54: 33-38.
Oliveira RAG, Oliveira KRA, Diniz MFFM 1997. A fitoterapia no serviço de saúde pública da Paraíba. Rev Extensão, João Pessoa 2: 21-31.

Oliveira FQ, Gobira B, Guimarães C, Batista J, Barreto M, Souza M 2007. Espécies vegetais indicadas na odontologia. Rev Bras Farmacogn 17: 466-476.

Ribeiro AQ, Leite JPV, Dantas-Barros AM 2005. Perfil de utilização de fitoterápicos em farmácias comunitárias de Belo Horizonte sob a influência da legislação nacional. Rev Bras Farmacogn 15: 65-70.

Semanário Oficial 1994. Município de João Pessoa 1994. Lei Ordinária $\mathrm{n}^{\circ} 7630$ de 15 de julho de 1994. Semanário Oficial $n^{\circ} 393 / 1994$ publicado no DOU em 19 de julho de 1994.

Silva MIG, Gondim APS, Nunes IFS, Sousa FCF 2006. Utilização de fitoterápicos nas unidades básicas de atenção à saúde da família no município de Maracanaú (CE). Rev Bras Farmacogn 16: 455-462.

Silva MSA, Silva MAR, Higino JS, Pereira MSV, Carvalho AAT 2008. Atividade antimicrobiana e antiaderente in vitro do extrato de Rosmarinus officinalis Linn. sobre bactérias orais planctônicas. Rev Bras Farmacogn 18: 236-240.

Singh J, Kumar A, Budhiraja S, Hooda A 2007. Ethnomedicine: use in dental caries. Braz J Oral Sci 6: 1308-1312.

Soyama P 2007. Plantas medicinais são pouco exploradas pelos dentistas. Cienc Cult 59: 12-13.

Vasconcelos LCS, Sampaio FC, Sampaio MCC, Pereira MSV, Higino JS, Peixoto MHP 2006. Minimum inhibitory concentration of adherence of Punica granatum Linn (pomegranate) gel against $S$. mutans, S. mitis and $C$. albicans. Braz Dent J 17: 223-227.

Vasconcelos KRF, Veiga Junior VF, Rocha WC, Bandeira MFCL 2008. Avaliação in vitro da atividade antibacteriana de um cimento odontológico à base de óleo-resina de Copaifera multijuga Hayne. Rev Bras Farmacogn 18 (Supl.): 733-738.

Veiga-Junior VF 2008. Estudo do consumo de plantas medicinais na Região Centro-Norte do Estado do Rio de Janeiro: aceitação pelos profissionais de saúde e modo de uso pela população. Rev Bras Farmacogn 18: 308-313.

Xavier MN, Ramos INC, Xavier-Filho L 1995. A fitoterapia no combate às afecções bucais. João Pessoa: ed. Idéia 75-101. 